1. De Wever O, Demetter P, Mareel M, Bracke M Stromal myofibroblasts are drivers of invasive cancer growth. Int J Cancer. 2008;123(10):2229-2238.

2. Finak G, et al. Stromal gene expression predicts clinical outcome in breast cancer. Nat Med. 2008; 14(5):518-527.

3. Bhowmick NA, Moses HL. Tumor-stroma interactions. Curr Opin Genet Dev. 2005;15(1):97-101.

4. Ishii $\mathrm{G}$, et al. Bone-marrow-derived myofibroblasts contribute to the cancer-induced stromal reaction. Biochem Biophys Res Commun. 2003;309(1):232-240.

5. McAllister SS, et al. Systemic endocrine instigation of indolent tumor growth requires osteopontin. Cell. 2008;133(6):994-1005.

6. Anborgh PH, Mutrie JC, Tuck AB, Chambers AF. Role of the metastasis-promoting protein osteopontin in the tumour microenvironment. J Cell Mol Med. 2010;14(8):2037-2044.

7. Elkabets $\mathrm{M}$, et al. Human tumors instigate granulin-expressing hematopoietic cells that promote malignancy by activating stromal fibroblasts in mice. J Clin Invest. 2011;121(2):784-799.

8. Randall TD, Weissman IL. Characterization of a population of cells in the bone marrow that phenotypically mimics hematopoietic stem cells: Resting cells or mystery population? Stem Cells. 1998;16(1):38-48.

9. Bateman A, Bennett HP. The granulin gene family: from cancer to dementia. Bioessays. 2009; 31(11):1245-1254.

10. Serrero G, Ioffe OB. Expression of PC-cell-derived growth factor in benign and malignant human breast epithelium. Hum Pathol. 2003;34(11):1148-1154.

11. Lu R, Serrero G. Inhibition of PC cell-derived growth factor (PCDGF, epithelin/granulin precursor) expression by antisense PCDGF cDNA transfection inhibits tumorigenicity of the human breast carcinoma cell line MDA-MB-468. Proc Natl Acad Sci U S A. 2000;97(8):3993-3998.

12. Ho JC, et al. Granulin-epithelin precursor as a therapeutic target for hepatocellular carcinoma. Hepatology. 2008;47(5):1524-1532.

13. He Z, Bateman A. Progranulin gene expres- sion regulates epithelial cell growth and promotes tumor growth in vivo. Cancer Res. 1999; 59(13):3222-3229.

14. Miyanishi $M$, et al. Immortalized ovarian surface epithelial cells acquire tumorigenicity by Acrogranin gene overexpression. Oncol Rep. 2007; 17(2):329-333.

15. He Z, Ong CH, Halper J, Bateman A. Progranulin is a mediator of the wound response. Nat Med. 2003;9(2):225-229.

16. Orimo A, et al. Stromal fibroblasts present in invasive human breast carcinomas promote tumor growth and angiogenesis through elevated SDF-1/ CXCL12 secretion. Cell. 2005;121(3):335-348.

17. Kaplan RN, et al. VEGFR1-positive haematopoietic bone marrow progenitors initiate the pre-metastatic niche. Nature. 2005;438(7069):820-827.

18. Holmgren L, O'Reilly MS, Folkman J. Dormancy of micrometastases: balanced proliferation and apoptosis in the presence of angiogenesis suppression. Nat Med. 1995;1(2):149-153.

\title{
Nuclear receptors take center stage in Th17 cell-mediated autoimmunity
}

\author{
Jennifer J. Heller, Ju Qiu, and Liang Zhou
}

Department of Pathology and Department of Microbiology and Immunology, Feinberg School of Medicine, Northwestern University, Chicago, Illinois, USA.

\begin{abstract}
Liver $X$ receptors (LXRs) are nuclear receptors involved in cholesterol homeostasis. Notably, they are also expressed by $T$ cells and are involved in regulating $\mathrm{T}$ cell proliferation and differentiation. In this issue of the JCI, Cui et al. have elucidated the molecular mechanism underlying the effects of LXR activation on a subset of T cells known as Th17 cells in mice and humans. Specifically, they showed that LXR-induced Srebp-1 inhibits Il17 transcription by binding to the Il17 promoter through interaction with the aryl hydrocarbon receptor (Ahr), a transcription factor known to enhance Th17 cell responses.
\end{abstract}

Heightened immune responses are needed to fight harmful microorganisms, yet those same responses have to be kept from overreacting and attacking the body's normal tissues to cause autoimmunity. This balance is maintained by a complex set of interactions among various immune cells. Th17 cells are a subset of $\mathrm{CD} 4^{+} \mathrm{T}$ cells characterized by the production of high levels of the cytokines IL-17 (also known as IL-17A), IL-17F, and IL-22 (1). They are important for the clearance of certain bacterial and fungal pathogens (1). However, Th17 cells have also been implicated in various human autoimmune diseases and other chronic inflammatory conditions (1). Since the initial discovery that the nuclear retinoic

Conflict of interest: The authors have declared that no conflict of interest exists.

Citation for this article: J Clin Invest. 2011;

121(2):519-521. doi:10.1172/JCI45939. acid-related orphan receptor $\gamma \mathrm{t}(\mathrm{ROR} \gamma \mathrm{t})$ is key to the differentiation of Th17 cells (2), transcriptional regulation of Th17 cells has been intensively investigated.

\section{Aryl hydrocarbon receptor, an important regulator of Th17 cell differentiation}

Aryl hydrocarbon receptor (Ahr), a liganddependent nuclear receptor, is among the handful of transcription factors implicated thus far in Th17 cell differentiation (3). Although its endogenous ligands remain elusive, Ahr is best known to mediate the effects of environmental toxins (e.g., the human carcinogen dioxin; ref. 4). Ahr belongs to the basic region helix-loop-helix (bHLH)/Per-Arnt-Sim (PAS) family of proteins (4). Ligand binding to the PAS domain triggers the transcriptional activity of Ahr, inducing target gene expression. Ligandactivated Ahr enhances the expression of IL-17 and is essential for IL-22 expression (5). Recent data suggest that Ahr may promote Th17 cell differentiation, in part through suppression of Stat1 phosphorylation (6). However, the precise molecular mechanism(s) by which Ahr regulates Th17 cell differentiation still remain elusive.

Role of liver $\mathrm{X}$ receptors in regulating immune response

Liver $\mathrm{X}$ receptors (LXRs) were originally classified as orphan nuclear receptors. However, it was subsequently determined that both LXR isoforms (LXR $\alpha$ and LXR $\beta$ ) are ligand-dependent transcription factors activated by metabolites of cholesterol that cause a conformational change in the LXR upon binding (7). LXRs form a heterodimer with retinoid $\mathrm{X}$ receptor (RXR), which then binds DNA and modulates the expression of various genes involved in regulating cholesterol homeostasis, including $A B C A 1, A B C G 1$, and SREBP1 (7). More recent studies have expanded the role of LXRs to implicate them as modulators of the immune response (8-11). LXR ligands have been shown to inhibit LPSor cytokine-induced expression of genes encoding inflammatory mediators such as IL-6 (8), and LXR-dependent gene expression is required for macrophage function in response to bacterial infection (9). 

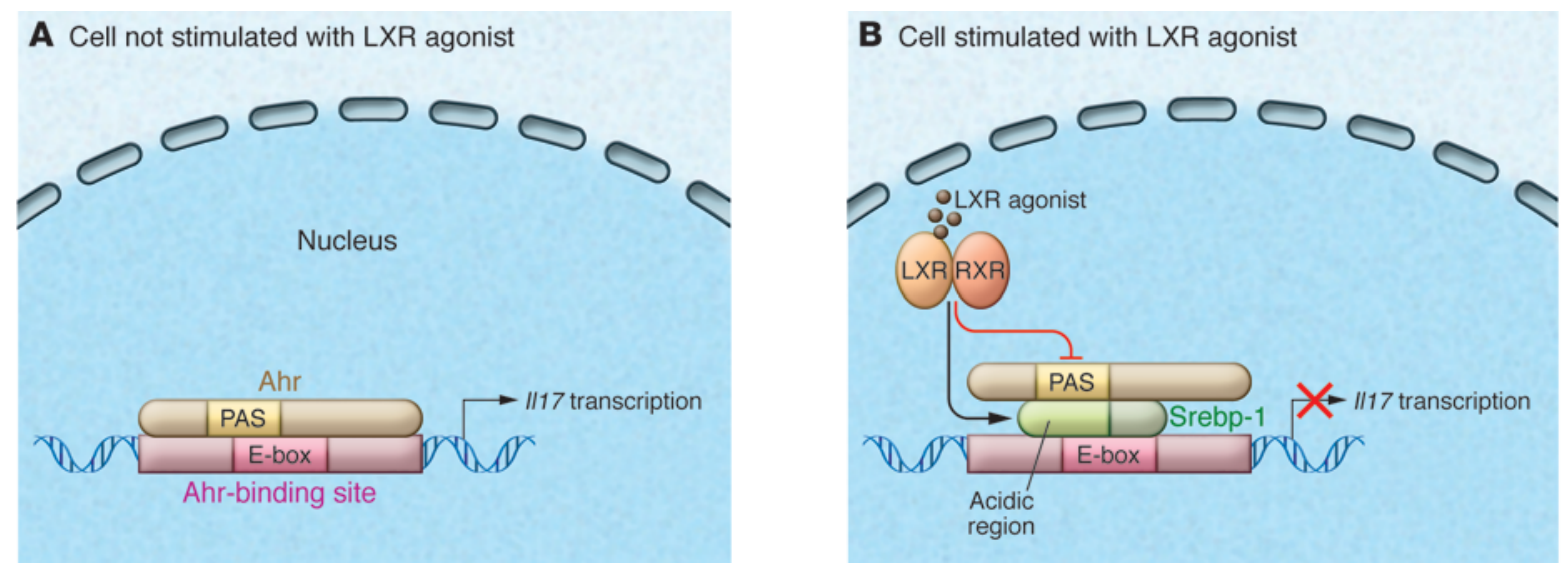

Figure 1

Molecular mechanisms underlying LXR-mediated negative regulation of IL-17 expression in Th17 cells. (A) In this issue of the JCl, Cui et al. report that Ahr, a ligand-dependent transcription factor, increases IL-17 expression by binding to the //17 promoter (13). (B) LXR activation by its agonists induces and activates Srebp-1. A putative binding site of Srebp-1 is found at the E-box element on the I/17 promoter that overlaps with the Ahrbinding site, suggesting that Srebp-1 competes with Ahr for binding to this region of the $/ / 17$ promoter to suppress the activating effects of Ahr on IL-17 expression. Srebp-1 interacts with the PAS domain of Ahr, subsequently blocking the enhancement of //17 transcription. LXR activation also inhibits the expression of other genes implicated in Th17 cell differentiation (e.g., Ahr).

Because both isoforms of LXR are expressed in $\mathrm{CD}^{+} \mathrm{T}$ cells (11), it was also thought that LXRs might play a role in the adaptive immune response. Indeed, it has been reported that LXR activation inhibits lymphocyte proliferation (10). Bensinger et al. showed that ligand activation of LXRs blocked mitogen-driven $T$ cell expansion by altering cellular sterol content via a pathway requiring the LXR target gene $A b c g 1$, whereas loss of LXR expression conferred a proliferative advantage to lymphocytes and an enhanced immune response (10). Additionally, it has been recently found that LXR activation and signaling are necessary for apoptotic cell clearance as well as the maintenance of immune tolerance by macrophages (12). Apoptotic cells activate LXRs, inducing expression of the receptor tyrosine kinase Mer, which is critical for phagocytosis (12). Mice deficient for both LXR $\alpha$ and LXR $\beta$ exhibit decreased self-tolerance and eventually develop autoimmune glomerulonephritis, a mouse model of lupus-like autoimmunity (12). This suggests that LXR activation blocks inflammatory immune responses by both inhibiting lymphocyte proliferation and preventing the initial exposure of self-reactive antigens to $\mathrm{T}$ cells by regulating apoptotic cell clearance.

\section{Ahr/LXR crosstalk in Th17 differentiation and autoimmunity}

In this issue of the JCI, Cui et al. report another novel role for LXR in negatively regulating mouse and human Th17 cell differentiation by disrupting Ahrinduced IL-17 production (13). Their data suggest that LXR-induced Srebp-1 suppresses Th17 differentiation by binding to the E-box element in the Il17 promoter that overlaps with an Ahr-binding site (Figure 1). Furthermore, they show that Srebp-1 physically interacts with Ahr and prevents Ahr binding to the DNA to induce $I l 17$ transcription.

A role for LXRs in regulating Th17 cells was initially suggested by earlier experiments showing that an LXR agonist, T0901317, was able to inhibit the development of EAE by suppressing expression of IL-23R and the Th17 cytokines IL-17, IL-21, and IL-22 (14). Since T0901317 also binds to other nuclear receptors (e.g., farnesoid X receptor, $\operatorname{ROR} \gamma$, and $\operatorname{ROR} \alpha$ ), the role of LXR in Th17 cell differentiation was unclear (15). The data published by Cui et al. provide evidence for a novel mechanism of LXR-mediated negative regulation of Th17 cell differentiation using elegant gain-of-function and loss-of-function approaches (13). Overexpression of LXR in naive $\mathrm{CD}^{+} \mathrm{T}$ cells inhibited differentiation to the Th17 lineage in a dosedependent manner, while a deficiency of both LXR isoforms promoted Th17 cell differentiation. These results were corroborated by in vivo studies in the EAE model of multiple sclerosis, where LXR agonists inhibited IL-17 secretion but not IFN- $\gamma$ secretion in the CNS, suggesting a selective role for LXRs in suppressing Th17 dif- ferentiation in vivo. These results were not unique to mice, as it was shown that LXR activation also decreased in vitro human Th17 cell differentiation. Cui et al. further showed that Srebp-1, which is encoded by a downstream target gene of LXR, exerted an inhibitory effect on the Il17 promoter to reduce $I l 17$ transcription after translocation into the nucleus following LXR activation (13). The Ahr-mediated activation of Il17 transcription was suppressed by LXR-activated Srebp-1, which inhibited Ahr activity through physical interaction (Figure 1). Besides inhibition of Ahr function, ligand activation of LXR also inhibited the expression of the nuclear receptors ROR $\gamma t$ and Ahr, which are implicated in driving Th17 cell differentiation. These findings provide another example of negative regulation of Th17 cell differentiation through protein-protein interaction in addition to the previously established inhibitory interaction between the transcription factor forkhead box protein 3 (Foxp3) and RORyt (16).

\section{Future studies of LXR-mediated negative regulation of Th17 cells}

The current study by Cui et al. (13) provides a satisfying molecular explanation for how ligand-activated LXR inhibits Th17 cell differentiation through negative interaction between Srebp-1 and Ahr, and thereby how it ameliorates autoimmunity (e.g., EAE). There are, however, several interesting questions that 
remain to be answered. First, the LXR agonist T0901317 has been found to potently inhibit the transactivation activity of ROR $\gamma$, an isoform of ROR $\gamma$ t that is expressed in non-hematopoietic cells, resulting in modulation of the ability of the receptor to interact with transcriptional cofactor proteins (15). Interestingly, ROR $\gamma$ has recently been shown to bind cholesterol derivatives (17). Since ROR $\gamma$ and ROR $\gamma$ t share the same ligand-binding domain (18), these results suggest that ROR $\gamma t$, much like LXR and ROR $\gamma$, may bind and perhaps respond to metabolites of cholesterol, providing a possible crosstalk between ROR $\gamma t$ and LXR. The link between cholesterol levels and autoimmune inflammation thus needs to be further explored. Another remaining question is, How does LXR inhibit the expression of other genes encoding factors implicated in Th17 cell differentiation and function such as IL-17F, IL-22, and IL-23R? It will be interesting to determine whether inhibition of these genes is due to interaction of Srebp-1 and Ahr at their promoters or is an indirect result of reduced ROR $\gamma t$ and Ahr levels. Cui et al. provide strong evidence that LXR agonists inhibit Ahr signaling (13). It remains to be determined whether the interaction of Srebp-1 through the PAS domain of Ahr could interfere with Ahr ligand binding, thus inhibiting the transcriptional activity of Ahr. Further, given that LXR antagonizes the activity of Ahr, it remains to be determined whether activation of Ahr could interfere with LXR signaling and/or activity.

\section{Clinical implications of LXR for Th17- mediated immunotherapy}

Although recent findings suggest that LXR would be a promising target for therapy in Th17 cell-mediated inflammatory diseases, it has been found to have varying effects on autoimmune disorders. Activation of LXR relieved EAE in an experimental model of multiple sclerosis, but worsened collagen-induced arthritis by aggravating articular inflammation and cartilage destruction (14, 19). The role of LXR in immunity, with both activating and suppressive functions, is an area of ongoing investigation. It is tempting to speculate that this dual function could be linked to the activity of
Ahr in these models. In EAE models, Ahr activation leads to earlier onset of disease and increased severity $(5,20)$. LXR agonists in this model can block the effects of Ahr, thus reducing CNS inflammation. Intriguingly, increased Ahr activation has also been shown to exacerbate RA symptoms (21), but LXR agonists do not relieve symptoms in collagen-induced arthritis mouse models; rather, they exacerbate the disease (19). This seemingly contradictory result could be due to a negative regulatory role of Ahr in certain immune cells (e.g., Tregs, macrophages, and dendritic cells). For example, some Ahr ligands have been proposed to promote Treg differentiation and thus may exert an immune-suppressive function $(4,20)$. In LPS-induced inflammatory responses, genetic ablation of $\mathrm{Abr}$ in mouse macrophages led to elevated levels of IL- 6 and hence increased sensitivity to endotoxin shock (22). Consistent with this, LXR activation led to augmented release of cytokines by monocytes obtained from RA patients (19). Most recently, Ahr has been identified as a negatively regulator of dendritic cell immunogenicity via a kynurenine-dependent mechanism (23). Together, these data imply that the inhibition of Ahr via LXR may have different biological outcomes depending on different cell types and disease models. Nevertheless, the findings of Cui et al. (13) have important implications for those seeking to develop treatments for Th17 cell-mediated inflammatory autoimmune diseases by targeting nuclear receptor pathways (e.g., those regulated by AHR, LXRs, and RORs).

\section{Acknowledgments}

This work was supported in part by NIH grant AI089954 (to L. Zhou).

Address correspondence to: Liang Zhou, 300 E. Superior Street, Tarry 3-762, Chicago, Illinois 60611, USA. Phone: 312.503.3182; Fax: 312.503.8240; E-mail: L-Zhou@northwestern.edu.

1. Korn T, Bettelli E, Oukka M, Kuchroo VK. IL-17 and Th17 cells. Annu Rev Immunol. 2009;27:485-517.

2. Ivanov II, et al. The orphan nuclear receptor RORgammat directs the differentiation program of proinflammatory IL-17+ T helper cells. Cell. 2006;126(6):1121-1133.

3. Zhou L, Littman DR. Transcriptional regulatory networks in Th17 cell differentiation. Curr Opin
Immunol. 2009;21(2):146-152.

4. Stevens EA, Mezrich JD, Bradfield CA. The aryl hydrocarbon receptor: a perspective on potential roles in the immune system. Immunology. 2009;127(3):299-311.

5. Veldhoen $M$, et al. The aryl hydrocarbon receptor links TH17-cell-mediated autoimmunity to environmental toxins. Nature. 2008;453(7191):106-109.

6. Kimura A, Naka T, Nohara K, Fujii-Kuriyama Y, Kishimoto T. Aryl hydrocarbon receptor regulates Stat 1 activation and participates in the development of Th17 cells. Proc Natl Acad Sci U S A. 2008; 105(28):9721-9726.

7. Zelcer N, Tontonoz P. Liver X receptors as integrators of metabolic and inflammatory signaling. J Clin Invest. 2006;116(3):607-614.

8. Joseph SB, Castrillo A, Laffitte BA, Mangelsdorf DJ, Tontonoz P. Reciprocal regulation of inflammation and lipid metabolism by liver $\mathrm{X}$ receptors. Nat Med. 2003;9(2):213-219.

9. Joseph SB, et al. LXR-dependent gene expression is important for macrophage survival and the innate immune response. Cell. 2004;119(2):299-309.

10. Bensinger SJ, et al. LXR signaling couples sterol metabolism to proliferation in the acquired immune response. Cell. 2008;134(1):97-111.

11 . Walcher $\mathrm{D}$, et al. LXR activation reduces proinflammatory cytokine expression in human CD4positive lymphocytes. Arterioscler Thromb Vasc Biol. 2006;26(5):1022-1028.

12. A-Gonzalez N, et al. Apoptotic cells promote their own clearance and immune tolerance through activation of the nuclear receptor LXR. Immunity. 2009;31(2):245-258.

13. Cui $G$, et al. Liver $X$ receptor (LXR) mediates negative regulation of mouse and human Th17 differentiation. J Clin Invest. 2011;121(2):658-670.

14. Xu J, Wagoner G, Douglas JC, Drew PD. Liver $\mathrm{X}$ receptor agonist regulation of Th17 lymphocyte function in autoimmunity. J Leukoc Biol. 2009;86(2):401-409.

15. Kumar N, et al. The benzenesulfonamide T0901317 [N-(2,2,2-trifluoroethyl)-N-[4-[2,2,2-trifluoro-1hydroxy-1-(trifluoromethyl)ethyl]phenyl]-benzenesulfonamide] is a novel retinoic acid receptor-related orphan receptor-alpha/gamma inverse agonist. Mol Pharmacol. 2010;77(2):228-236.

16. Zhou L, et al. TGF-beta-induced Foxp3 inhibits $\mathrm{T}(\mathrm{H}) 17$ cell differentiation by antagonizing RORgammat function. Nature. 2008;453(7192):236-240.

17. Solt LA, Griffin PR, Burris TP. Ligand regulation of retinoic acid receptor-related orphan receptors: implications for development of novel therapeutics. Curr Opin Lipidol. 2010;21(3):204-211.

18. Eberl G, Littman DR. The role of the nuclear hormone receptor RORgammat in the development of lymph nodes and Peyer's patches. Immunol Rev. 2003;195:81-90.

19. Asquith DL, et al. Liver X receptor agonism promotes articular inflammation in murine collagen-induced arthritis. Arthritis Rheum. 2009;60(9):2655-2665.

20. Quintana FJ, et al. Control of T(reg) and T(H)17 cell differentiation by the aryl hydrocarbon receptor. Nature. 2008;453(7191):65-71.

21. Kobayashi S, et al. A role for the aryl hydrocarbon receptor and the dioxin TCDD in rheumatoid arthritis. Rheumatology (Oxford). 2008;47(9):1317-1322.

22. Kimura A, et al. Aryl hydrocarbon receptor in combination with Stat 1 regulates LPS-induced inflammatory responses. J Exp Med. 2009;206(9):2027-2035.

23. Nguyen NT, et al. Aryl hydrocarbon receptor negatively regulates dendritic cell immunogenicity via a kynurenine-dependent mechanism. Proc Natl Acad Sci US A. 2010;107(46):19961-19966. 